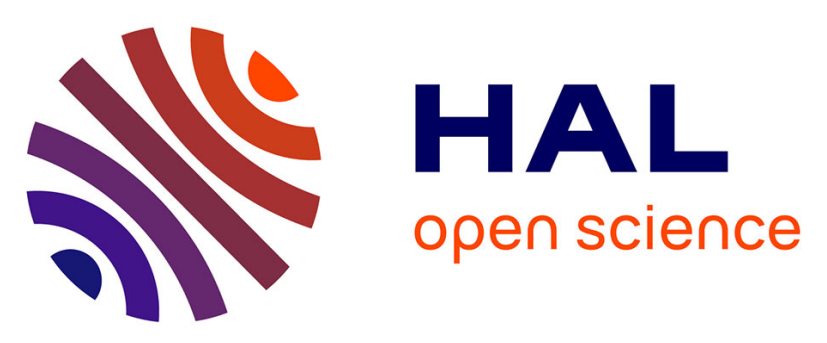

\title{
Plastic deformation by conservative shear-coupled migration of tilt boundaries with intergranular nano-cracks or precipitates
}

Aitor Luque, Javier Aldazabal, José Manuel Martínez-Esnaola, Javier Gil Sevillano

\section{To cite this version:}

Aitor Luque, Javier Aldazabal, José Manuel Martínez-Esnaola, Javier Gil Sevillano. Plastic deformation by conservative shear-coupled migration of tilt boundaries with intergranular nano-cracks or precipitates. Philosophical Magazine, 2010, 90 (27-28), pp.3617-3632. 10.1080/14786430903097715. hal-00590602

\section{HAL Id: hal-00590602 https://hal.science/hal-00590602}

Submitted on 4 May 2011

HAL is a multi-disciplinary open access archive for the deposit and dissemination of scientific research documents, whether they are published or not. The documents may come from teaching and research institutions in France or abroad, or from public or private research centers.
L'archive ouverte pluridisciplinaire HAL, est destinée au dépôt et à la diffusion de documents scientifiques de niveau recherche, publiés ou non, émanant des établissements d'enseignement et de recherche français ou étrangers, des laboratoires publics ou privés. 


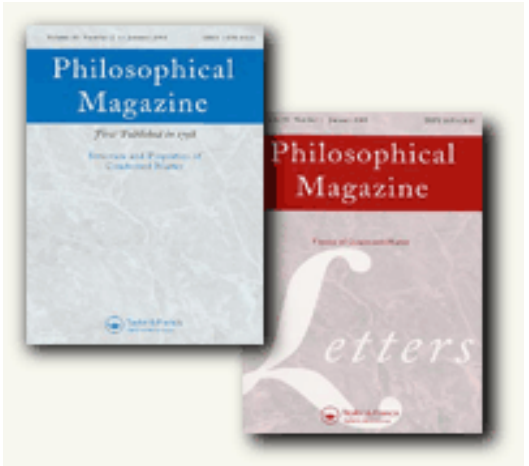

\section{Plastic deformation by conservative shear-coupled migration of tilt boundaries with intergranular nano-cracks or precipitates}

\begin{tabular}{|c|c|}
\hline Journal: & Philosophical Magazine \& Philosophical Magazine Letters \\
\hline Manuscript ID: & TPHM-09-Mar-0089.R1 \\
\hline Journal Selection: & Philosophical Magazine \\
\hline $\begin{array}{l}\text { Date Submitted by the } \\
\text { Author: }\end{array}$ & 19-May-2009 \\
\hline Complete List of Authors: & $\begin{array}{l}\text { Luque, Aitor; CEIT and TECNUN (University of Navarra), Materials } \\
\text { Department } \\
\text { Aldazabal, Javier; CEIT and TECNUN (University of Navarra), } \\
\text { Materials Department } \\
\text { Martínez-Esnaola, José Manuel; CEIT and TECNUN (University of } \\
\text { Navarra), Materials Department } \\
\text { Gil Sevillano, Javier; CEIT and TECNUN (University of Navarra), } \\
\text { Materials Department }\end{array}$ \\
\hline Keywords: & boundary migration, grain boundaries, molecular dynamics \\
\hline Keywords (user supplied): & $\begin{array}{l}\text { shear-coupled migration, stress driven migration, intergranular } \\
\text { nano-cracks }\end{array}$ \\
\hline
\end{tabular}

\section{S) ScholaroNE \\ Manuscript Central}




\title{
Plastic deformation by conservative shear-coupled migration of tilt boundaries with intergranular nano-cracks or precipitates
}

\author{
A. LUQUE, J. ALDAZABAL, J.M. MARTÍNEZ-ESNAOLA, J. GIL SEVILLANO**
}

\begin{abstract}
We present molecular dynamics (MD) simulations of the shear-coupled migration (SCM) behaviour of symmetrical tilt boundaries perturbed by the presence of nano-cracks or nano-precipitates lying on the boundary plane. The simulations have been performed for copper bicrystals at room temperature $(300 \mathrm{~K})$. The tilt boundary gets pinned by the crack tip or by the precipitates; shear-coupled migration occurs only ahead of the pinning points. Bulging of the tilt boundary reduces the shear stress on the boundary surface near the pinning points. In the case of cracks the local deviation of the boundary from the crack plane close to the crack tip hinders mode II crack propagation; in fact, some crack healing is observed in some cases. The applied stress grows until depinning of the boundary takes place by SCM bulging or by the combined action of SCM with another deformation mechanism (emission of dislocations from the pinning point vicinity, grain boundary sliding).
\end{abstract}

Keywords: grain boundaries, shear-coupled migration, stress driven migration, tilt grain boundaries, molecular dynamics, intergranular nano-cracks.

* Corresponding author: jgil@ ceit.es

\section{Introduction}

First references to grain boundary (GB) movement as a consequence of applied mechanical loads or forced external displacements date back to the 1950's for small-angle tilt boundaries [1, 2] or mainly to the 1970's or 1980's for high-angle GBs [3-7]. Only recently this phenomenon has started to attract much attention and to be systematically studied either through experiments [813], or theoretically [14] or by numerical simulations [15-20]. We refer here to stress/strain coupled GB migration (limited in fact to shear-coupled migration of tilt boundaries or associated to the tilt component of grain boundaries), not to be mistaken with "strain-induced GB migration", a term used since early recrystallization studies to denote the preferred migration of a GB separating two grains with a very different strain-induced dislocation density [21, 22].

Shear-coupled migration (SCM) of tilt boundaries has been acknowledged as a particular plastic strain mechanism that can, in a wide temperature range, complement or compete with the other plastic intra-or inter-granular mechanisms available to bicrystals or polycrystals (lattice dislocation-mediated crystallographic slip, grain boundary sliding, twinning, etc.). Conservative SCM of pure tilt boundaries, diffusionless but thermally activated, can occur at low temperatures and still be the dominant shear-driven GB migration mechanism up to very high temperatures relative to the melting point, at least for high shear strain rates [16, 19], before a cross-over to viscous-like creep process takes place. It occurs by collective atomic motion without recourse to 
long-range diffusion. The current burst of interest on SCM is due to its active role in the mechanical behaviour of nanograined materials and in the structural changes taking place in them under stress at low temperatures. Conservative SCM requires high resolved shear stresses that cannot be reached in polycrystals with conventional grain size before attaining the activation stress for profuse dislocation glide. Observance of the Hall-Petch relationship is the signature for dominance of dislocation glide or, more generally, of low-temperature intragranular plastic mechanisms over intergranular plastic processes. At room temperature for instance, inversion of the Hall-Petch effect is observed in copper for an equivalent tensile yield stress of approx. $0.9 \pm 0.3 \mathrm{GPa}$ [23] and according to molecular dynamics (MD) simulations [16,19 and this paper] $\mathrm{SCM}$ requires resolved shear stresses ranging from $0.25 \mathrm{GPa}$ to $0.4 \mathrm{GPa}$, equivalent to tensile stresses of the order of the above limit tensile yield stress. Thus, this mechanism will only be relevant for nanograined copper. Moreover, SCM is believed to be the main cause of the grain coarsening observed to take place at low temperatures in nanograined polycrystals under stress: grain growth under nanoindentations, near loaded crack tips or during further plastic deformation processing or fatigue of nanomaterials [24-35]. Numerical MD simulations support such observations [36-40]. A good understanding and mastering of SCM in nanograined materials is thus essential in order to warrant their structural stability.

The shear strain effectiveness of the migration of a tilt boundary is characterized by a shear coupling factor, $\beta$, the ratio of the shear displacement parallel to the GB surface in the direction normal to the tilt axis, $v_{s}$, to the GB migration normal to its surface, $v_{n}$, i.e., $\beta$ is the shear strain associated to the passage of the migrating boundary:

$\beta=\frac{v_{s}}{v_{n}}=\Gamma_{S C M}$

The macroscopic plastic strain associated to SCM is given by:

$d \bar{\varepsilon}=\beta M_{S C M} d\left(V_{V}\right)_{S C M}$

where $M_{S C M}$ is an orientation factor and $\left(V_{V}\right)_{S C M}$ the volume fraction swept by the migrating boundary. Notice that thickening or thinning (detwinning) of mechanical twins is a very particular case of SCM, with $\beta$ the twinning shear strain ( $\sqrt{2} / 2$ for FCC materials). Also, grain boundary sliding (GBS) can be viewed as the limit case of SCM, when $\beta \rightarrow \infty$. Like GBS or pure diffusional deformation, SCM is not a self-sufficient plastic mechanism for arbitrary deformation of polycrystals, it always needs to be complemented by the activity of other deformation mechanisms in order to provide compatible large deformations of grain aggregates.

The factor $\beta$ is purely geometrical, determined by the tilt GB misorientation, $\theta$. For instance, for symmetrical coincident $<010>$ tilt boundaries in cubic bicrystals, either $\beta=2 \tan (\theta / 2)$ (positive coupling, general $\beta$ value for "small" misorientations) or $\beta=-2 \tan (\varphi / 2)$, $\varphi=(\pi / 2)-\theta$ (negative coupling, "large" misorientations), the transition taking place at $\theta \approx 35^{\circ}$ $[16,17]$. The coupling factor is related to the tilt misorientation through the Frank-Bilby vector 
(the net dislocation content of the GB), the GB migration taking place by slip of grain boundary dislocations (GBD) derived from Frank-Bilby's equation [41]: <100> dislocations for low misorientation, $<101>$ dislocations for high misorientation, cf. fig. 1. However, the Frank-Bilby equation yields a multiplicity of geometrically possible coupling factors, i.e., there is a multiplicity of $\beta$ values for the bicrystal to select (from the structural point of view, a multiplicity of geometrically possible GBD sets). For special (near-coincident) boundaries those sets are perfect dislocations of the crystal lattice and the DSC (displacement shift complete) lattice. For general non-coincident boundaries the situation is more complicated, because a coupling exclusively based on glide of GBD compatible with the lattices is impossible: the migration of the boundary implies atom shuffling besides the shear transformation responsible for the SCM deformation [33]. With respect to the spatial orientation of the GB plane relative to the reference frame defined by the shear plane and direction, different coupling modes (different $\beta$ values) are selected for different shear directions on the GB plane [42]. One could expect that changing the inclination of the GB plane about the tilt axis would also lead to different coupling modes, ref. [20] presents evidence of this for a general tilt boundary; the point is of interest for the SCM of curved boundaries: a different $\beta$ in two segments of a migrating boundary would imply the trailing of a wall of geometrically necessary defects in order to accommodate the strain heterogeneity.

According to Mishin and co-workers [16, 18, 19] the kinetics of conservative SCM is of stickslip type and responds to the equation

$v_{s}=\frac{\tau_{c}^{0}}{\eta}\left(1-\frac{\tau_{c}}{\tau_{c}^{0}}\right)^{1 / 2} \exp \left[-\frac{\left(1-\tau_{c} / \tau_{c}^{0}\right)^{3 / 2} E^{0}}{k T}\right]$

where $\tau_{c}$ is the resolved shear stress acting on the GB and $k$ the Boltzmann constant. The material parameters $\tau_{c}^{0}, \eta$ and $E^{0}$ depend on both the misorientation of the tilt $\mathrm{GB}$ and the inclination of its plane. The critical shear stress for a given shear rate is typically smaller for symmetrical inclinations of the GB plane [20], a fact probably linked to their higher atomic density.

In this work we present results of MD simulations of shear-coupled migration of several symmetrical tilt boundaries in copper at room temperature. The simulations were aimed to compare the SCM behaviour of perfect boundaries with that of boundaries containing nanocracks or nano-precipitates lying on their surface. Intergranular nano-defects are frequent in nanograined materials and it is generally assumed that they are inherited from their processing. It has been suggested that they could be inherent to such materials [43]. The objective of our work was to explore the possible stabilization of nanograined structures by a natural or artificial nanodefect population (a practical connection could be the SCM behaviour of radiation-damaged nanostructured materials). In the case of cracks, the mode II stress field near the crack tip was expected to modify the migration response of the GB although it could also modify the local activity of the other deformation mechanisms: dislocation emission, GB sliding. In the case of nano-precipitates, SCM was expected to be hindered by the pinning action of the precipitates on the boundaries. 


\section{Simulation technique, specimens and tests}

Details of the MD technique, the embedded atom method and the copper potential employed in the simulations are given elsewhere [44]. The Nosé-Hoover thermostat [45] was implemented for temperature control. Simulations have been carried out at $T=300 \mathrm{~K}$ using time increments $\Delta t=$ $2.5 \times 10^{-15} \mathrm{~s}$.

The response of three high-coincidence symmetrical [001] tilt boundaries, $\Sigma 5$ (310), $\Sigma 17$ (530) and $\Sigma 41$ (540) has been studied (the Miller indices of the boundary planes are indicated besides the coincidence index $\Sigma$ ), see Table 1 . Notice that the tilt angle $\theta$ reported in the table corresponds to constructing the bicrystal by a positive rotation around [001] to bring the lower crystal in coincidence with the upper one. The true (minimum) tilt misorientations are $36.9^{\circ}$ for the $\Sigma 5$ boundary, $28.1^{\circ}$ for the $\Sigma 17$ one and $12.7^{\circ}$ for the $\Sigma 41$ one; the latter is thus a limit case of low-angle boundary (low-angle boundaries, constituted of lattice dislocations, occur for misorientations up to $10^{\circ}$ to $15^{\circ}$ ).

Prior to virtual testing of the samples, the cracked or uncracked bicrystals were constructed at 0 $\mathrm{K}$. Periodic boundary conditions were set along the $x$ and $y$ axes, axis $y$ being normal to the GB and axis $z$ being the [001] tilt axis (the dimensions of the repeating periodic parallelepipedic unit are given in Table 1). Periodic intergranular cracks across the section were formed by removing the atoms located in a band of $0.55 \mathrm{~nm}$ centred in the GB along 1/3 of the periodic unit size in the $x$ direction (the cut-off radius of the atom interactions was $0.55 \mathrm{~nm}$ ). Strong intergranular precipitates were formed by substituting the $\mathrm{Cu}$ atoms in the above referred band by atoms of a much more rigid material (the interatomic potential between precipitate atoms or between $\mathrm{Cu}$ precipitate atoms is ten times stiffer than the $\mathrm{Cu}-\mathrm{Cu}$ potential). For the $\mathrm{GB}$ to acquire its metastable configuration the bicrystals were relaxed under no constraints during $5 \mathrm{ps}$ at $0 \mathrm{~K}$ and along a linear temperature increase from $0 \mathrm{~K}$ to $300 \mathrm{~K}$ during $7 \mathrm{ps}$, followed by a relaxation at $300 \mathrm{~K}$ during $12.5 \mathrm{ps}$.

After relaxation, two rigid zones $0.55 \mathrm{~nm}$ thick were established in the upper and lower layers of the samples. During the virtual shear test, the lower rigid zone remained fixed, the upper part being horizontally displaced at a constant rate of $2.12 \mathrm{~ms}^{-1}(\Sigma 41, \Sigma 17)$ or $2.85 \mathrm{~ms}^{-1}(\Sigma 5)$ equivalent to a mean shear strain rate of the samples of about $10^{8} \mathrm{~s}^{-1}$. The height remained fixed during the shear. Periodic displacement boundary conditions were set along the $x$ and $y$ axes. The applied shear force and the imposed displacement are stored during the simulations, in order to compute the shear stress - shear strain curves. Atomic positions are also periodically stored for analyzing any structural changes. Atomistic configurations are viewed using the AtomEye software [46].

\section{Results}

All three GB tested showed negative shear coupling in agreement with published results that report negative coupling for $\theta \geq 35^{\circ}$ in [001] tilt boundaries. The values of the coupling factors 
agree with the Cahn-Mishin-Suzuki values [16]. Figure 2 shows the nominal shear stress, $\tau_{\text {nom, }}$ calculated as the sum of shear forces applied to the upper rigid layer along the $x$ axis divided by the layer area, as a function of the nominal shear strain, $\gamma_{\text {nom. }}$. The latter is given by the ratio of the horizontal displacement of the upper rigid layer to the height of the bicrystal. The average shear stress in the ligament of the cracked specimens is 1.5 times higher than the nominal shear stress.

For the uncracked or precipitate-free tilt boundaries (fig. 2a), the results reproduce the behaviour observed at similar homologous temperature by other authors [16-20], characterized by a stickslip phenomenon associated to the boundary migration when a certain threshold shear stress $(\approx$ $0.3 \mathrm{GPa}$ ) is overcome. The slopes of the intermittent elastic loading stages are $37.3 \mathrm{GPa}, 28.4$ GPa and 27.3 GPA for respectively the $\Sigma 5, \Sigma 17$ and $\Sigma 41$ boundaries, in good agreement with the values of the shear moduli (respectively, 41.6 GPa, 27.3 GPa and 23.9 GPa) calculated from the elastic constants of the $\mathrm{Cu}$ crystal [47].

The presence of an intergranular crack or precipitate significantly affects the mechanical response of the samples, cf. fig. $2 \mathrm{~b}$. The defective boundaries show similar stick-slip behaviour as perfect boundaries but the shear stress increases monotonically with strain till values of 1 to $1.5 \mathrm{GPa}$ at about $\gamma_{\text {nom }} \approx 0.1$, where a sudden big stress drop occurs, followed by successive stages of stress grow interspersed with stress drops at peaks never reaching the level of the first one.

This is at first sight a surprising result for cracked specimens, the bicrystal being strengthened by the presence of intergranular nanocracks, although the situation is similar to the strengthening of a crystal by a dispersion of nanovoids in dislocation-mediated plasticity [48, 49]. The shear strain-induced structural changes explain this "anomalous" behaviour: the cracks pin the grain boundaries and SCM is hindered. The unperturbed GBs migrate under shear stress retaining their structure and spatial orientation, cf. fig. 3. In a cracked bicrystal with a GB of intermediate $|\beta|$ value, like $\Sigma 17$, although the crack presence amplifies the shear stress in the vicinity of the tip, the GB is strongly pinned by the crack. The GB bows out because shear coupled migration only occurs away from the crack tip (downwards in this case, $\beta<0$ ). The shear stress applied on the GB surface close to the crack tip weakens because of the progressive GB deviation from its original plane; migration of the GB becomes progressively more difficult there because of the deviation of the boundary from the maximum shear plane. Crack propagation is hindered also for the same reason and because crack kinking would be necessary now for crack propagation. The applied stress rises until GB depinning from the crack occurs, accompanied of partial dislocation emission from the crack tip, fig. 4. This behaviour is observed for $\Sigma 41$ too (small $|\beta|$ ), fig. 5. For large $|\beta|$ values $(\Sigma 5)$, the GB is very stiff, the stress rises without much bowing and grain boundary sliding (GBS) occurs without significant SCM, cf. fig. 6. The GBS locally releases stress and produces the observed applied stress drop; GBS leads to some mode-II crack propagation. Quite similar behaviours are observed when the GBs contain intergranular precipitates instead of intergranular cracks. Of course the behaviour could be quite different in materials with intrinsically brittle or embrittled grain boundaries. 


\section{Discussion}

In our simulations we have seen that any interruption of the continuity of the tilt boundary by precipitates, nanocracks (or other GBs in the triple junctions of a GB network, [50]) hampers the shear-driven boundary migration. This is perhaps interesting for the stabilization of nanostructured materials, particularly ductile nanostructured materials, for which the presence of intergranular nanoprecipitates or nanocracks does not seem to induce a loss of toughness. This would be of interest, for instance, for the safe use of nanostructured materials under irradiation conditions generating defects (vacancy clusters) [51]. The obstruction for SCM is due to the pinning of a GB by its discontinuities (free surfaces [52], crack tips, interfaces).

Another point of interest for a discussion is that the effect of interactions of the migrating tilt boundary with cracks, precipitates, etc. will be dependent on their size and spacing. A shear stress $\tau$ applied on a flat tilt GB with a shear-coupling factor $\beta$ induces on it a virtual pressure

$P=\beta \tau$

Under a macroscopic shear stress $\tau$ applied on a free-to-move flat tilt GB (like in figs. 1 and 2), migration will start for a critical pressure $P_{c}=\beta \tau_{c}$. The critical threshold stress for SCM, given by eq. (3), can be viewed as the Peierls resolved shear stress for slip at the imposed rate of the particular GBDs constituting the boundary. From our results and results from the literature, its order of magnitude is $0.3 \mathrm{GPa}$. One can notice that, on account of typical values of the coupling factor, $\beta$ (Table 1), the critical pressure for SCM is also of the order of $0.3 \mathrm{GPa}$, much higher than the driving pressures for recrystallization or grain growth [53, 54]. Now, if pinned by cracks, precipitates, etc., spaced at a distance $L$ along the boundary, the GB needs to bow-out as it advances and such bow-out requires to apply an over-pressure above $P_{c}$ that will be dependent on the length $L$. If, in a too naïve analogy, the migrating GB is treated as a bulging membrane with a uniform specific surface energy $E_{S}$, the equilibrium migration condition should correspond (in our 2-D approximation) to the shape of an arc of circle of radius $R$,

$$
R=\frac{E_{S}}{P-P_{c}}=\frac{E_{S}}{\beta\left(\tau-\tau_{c}\right)}
$$

A limit condition for depinning would be reached when the critical condition $2 R=L$ is attained provided that no other deformation mechanisms intervenes before,

$$
\tau_{\text {depinning }} \leq \tau_{c}+\frac{2 E_{S}}{\beta L}
$$

The shear stress-strain behaviour of such a bicrystal of width $L$ with the GB pinned at its free surfaces can be obtained from eq. (5), on account of the volume swept by the bowing GB. It is depicted in fig. $7 \mathrm{a}$, assuming for the three boundaries a specific surface energy $E_{S}=1 \mathrm{Jm}^{-2}$ and the coupling factors of the symmetrical boundaries (Table 1). The initial slopes are proportional 
to $E_{S} \beta^{-2}$ (to be precise, the slope is $12 h w E_{S} /\left(\beta^{2} L^{3}\right)$, where $h$ and $w$ are the height and width of the sample, respectively) and the stress limit corresponds to eq. (6). For the latter, an inverse proportionality with the GB size $L$ is predicted. However, comparing with fig. $7 \mathrm{~b}$, it is clear that the naïve approximation is not adequate: although the order of magnitude is correct, neither the slopes nor the maximum stresses are well reproduced. This is not surprising: the shape of a twodimensional GB segment with fixed ends advancing in equilibrium is not a circular arc. A simple geometrical analysis based on the SGM mobilization of a flat tilt GB inclined at an angle $\alpha$ with respect to the macroscopic shear plane (fig. 8a) indicates that the driving pressure for free-end simple shear under a macroscopic shear stress $\tau$ is given by

$$
P_{\alpha}=\tau \beta_{\alpha} \cos ^{2} \alpha
$$

The coupling factor $\beta_{\alpha}$ is the shear strain (referred to the advancing boundary reference system) produced in the area swept by the advancing boundary of inclination $\alpha$ relative to the external shear plane. Consequently (fig. 8b), the radius of curvature of a two-dimensional GB advancing in equilibrium by SCM depends on the inclination of the boundary as

$$
\begin{aligned}
& R_{\alpha}=\frac{\left(E_{S}\right)_{\alpha}+\frac{\partial^{2}\left(E_{S}\right)_{\alpha}}{\partial \alpha^{2}}}{\beta_{\alpha}\left[\tau-\frac{\left(\tau_{c}\right)_{\alpha}}{\cos 2 \alpha}\right] \cos ^{2} \alpha}=\frac{\left(E_{S}\right)_{\alpha}+\frac{\partial^{2}\left(E_{S}\right)_{\alpha}}{\partial \alpha^{2}}}{\beta_{\alpha}\left[\tau \cos 2 \alpha-\left(\tau_{c}\right)_{\alpha}\right]}\left(1-\tan ^{2} \alpha\right) \\
& \tau \geq \frac{\left(\tau_{c}\right)_{\alpha}}{\cos 2 \alpha}
\end{aligned}
$$

where the specific energy dependence of the boundary segment on inclination has been considered [41, 55-58]. The geometry of the stressed grain boundary is analogous to that of the shape of a mixed dislocation under a resolved shear stress on its slip plane [59-63]. Equation (5) is only recovered for the $\alpha \rightarrow 0$ limit and neglecting the dependence of the GB specific energy on inclination. Although the shape of the stress-driven bulging GB is not semicircular, the size dependence of the critical stress for boundary depinning by reaching some unstable shape, with or without activation of other mechanism different from SCM, is still an inverse proportionality. Regarding the shape of the migrating GB, notice that there is a cusp for the inclination $\alpha=\pi / 4$. The optimal shape of the advancing tilt GB can be faceted on account of the influences of boundary spatial orientation on both the specific surface energy and virtual pressure for SCM.

Coming back to fig. $8 \mathrm{a}$, one can notice that the shear of the area swept by a migrating boundary segment inclined by $\alpha \neq 0$ with respect to the external shear plane implies extension and contraction deformations in the directions normal and parallel to the boundary in the plane of the figure. Under constrained conditions (imposed external displacement conditions, e.g., fixed-end simple shear) the irreversible SCM of a curved GB needs the simultaneous activation of other mechanisms (e.g., dislocation glide) in order to compensate for undesired strain components. In the simulations presented in this paper, some bulging of initially flat boundaries occurs by shear- 
coupled migration with elastic accommodation until dislocation emission or GBS are activated, absorb the misfit and relax the elastic stresses. A macroscopic stress drop is then observed and a new stage of SCM of the elastically accommodated curved GB occurs until activation of another mechanisms takes place again and so on. The macroscopic stress-strain curves of pinned boundaries migrating by SCM are thus serrated, each peak marking a relaxation episode corresponding to the activation of mechanisms other than SCM.

Finally, at the introduction of this paper we have suggested that SCM could play a decisive role in the breakdown of the Hall-Petch law and in the inversion of the grain size dependence experimentally observed in polycrystals below some critical grain size. For many tilt boundaries SCM activation requires smaller critical stresses than GBS [20]. At the critical stress for SCM of free flat boundaries, SCM of tilt GBs will start to contribute to plastic deformation. We have noted that such threshold stress for SCM is of the order of the shear stress for which the HallPetch breakdown is observed. Below some critical grain size, SCM will be irrelevant; however, for grain sizes smaller than the size for which the yield stress given by the Hall-Petch law equals the threshold stress for SCM, there is a finite stress-strain range for SCM activity: yielding will take place at a stress level below that expected from the Hall-Petch law (fig. 9). Moreover, as SCM leads to grain growth, softening (Hall-Petch inversion) is ensured. The relevance of SCM for plastic deformation once the SCM threshold stress is reached finds support in a recent statistical study of the GB character in cubic materials: in a random set of boundaries the proportion of near-tilt boundaries (capable of SCM) is strikingly large compared to the fraction of near-twist boundaries [64].

\section{Conclusions}

- Shear-coupled migration of symmetrical tilt boundaries is hindered by the presence of intergranular nanocracks.

- The tilt boundary is pinned by the crack tip and migration occurs with boundary bulging. The applied stress grows above the value needed for the migration of a perfect flat boundary until either depinning or grain boundary sliding occurs. The emission of lattice dislocations from the crack tip vicinity is simultaneous to shear-coupled migration of a constrained boundary and to the associated processes of depinning or boundary shear.

- Similar effects are observed if the continuity of the GB is broken by precipitates or by other disturbances of the bi-crystallinity (e.g., triple junctions in a polycrystalline structure).

- Shear-coupled migration may play a decisive role in the breakdown of the Hall-Petch relationship for very small grain sizes.

\section{Acknowledgements}

This work was supported by the Department of Industry, Commerce and Tourism of the Basque Government (project ETORTEK inanoGUNE). A. Luque also acknowledges the Spanish Ministry of Science and Innovation and the European Social Fund (Torres Quevedo Programme). 


\section{References}

[1] J. Washburn, E.R. Parquer, J. Metals-AIME Trans. 4 (1952) p. 1076.

[2] C.H. Li, E.H. Edwards, J. Washburn, E.R. Parker, Acta Metall. 1 (1953) p. 223.

[3] D. McLean, Nature 172 (1953) p. 300.

[4] S.G. Khayutin, Phys. Met. Metallogr. 37 (1974) p. 161.

[5] M. Guillopé, J.P. Poirier, Acta Metall. 28 (1980) 163.

[6] W.D. Means, M.W. Jessell, Tectonophysics 127 (1986) 67.

[7] M.W. Jessell, J. Struct. Geology 8 (1986) 527.

[8] M. Winning, G. Gottstein, L.S. Shvindlerman, Acta Mater. 49 (2001) p. 211.

[9] M. Winning, G. Gottstein, L.S. Shvindlerman, Acta Mater. 50 (2002) p. 353.

[10] M. Winning, Acta Mater. 51 (2003) p. 6465.

[11] S. Zaefferer, J.C. Kuo, Z. Zhao, M. Winning, D. Raabe, Acta Mater. 51 (2003) p. 4719.

[12] [14] M. Winning, A.D. Rollett, Acta Mater. 53 (2005) p. 2901.

[13] S. Badirujjaman, X.W. Li, M. Winning, Mater. Sci. Eng. A 448 (2007) p. 242.

[14] J.W. Cahn, J.E. Taylor, Acta Mater. 52 (2004) p. 4887.

[15]F. Sansoz, J.F. Molinari, Acta Mater. 53 (2005) p. 1931.

[16] J.W. Cahn, Y. Mishin, A. Suzuki, Acta Mater. 54 (2006) p. 4953.

[17] J.W. Cahn, Y. Mishin, A. Suzuki, Philos. Mag. 86 (2006) p. 3965.

[18] Y. Mishin, A. Suzuki, B.P. Uberuaga, A.F. Voter, Phys. Review B 75 (2007) p. 22401.

[19] V.A. Ivanov, Y. Mishin, Phys. Review B 78 (2008) p. 064106.

[20] H. Zhang, D. Dhu, D.J. Srolovitz, Philos. Mag. 88 (2008) p. 243.

[21] P.A. Beck, P.R. Sperry, J. Appl. Phys. 21 (1950) p. 150.

[22] P.A. Beck, Metal Interfaces, ASM, Cleveland, 1952 p. 208.

[23] Meyers, J. Metals 58 (2006) p. 41.

[24] M. Jin, A.M. Minor, E.A. Stach, J.W. Morris Jr., Acta Mater. 52 (2004) p. 5381.

[25] K. Zhang, J.R. Weertman, J.A. Eastman, Appl. Phys. Lett. 85 (2004) p. 5197.

[26] H. Mughrabi, H.W. Hoppel, M. Kautz, Scripta Mater. 51 (2004) 807.

[27] K. Zhang, J.R. Weertman, J.A. Eastman, Appl. Phys. Lett. 87 (2005) p. 061921.

[28] H. Van Swygenhoven, J.R. Weertman, Mater. Today 9 (2006) p. 24.

[29] G.J. Fan, L.F. Fu, D.C. Quiao, H. Choo, P.K. Liaw, N.D. Browning, Scripta Mater. 54 (2006) p. 2137.

[30] D.S. Gianola, D.H. Warner, J.F. Molinari, K.J. Hemker, Scripta Mater. 55 (2006) p. 649.

[31] X.Z. Liao, A.R. Kilmanetov, R.Z. Valiev, H. Gao, X. Li, A.K. Mukherjee, J.F. Bingert, Y.T. Zhu, Appl. Phys. Lett. 88 (2006) p. 021909.

[32] P.L. Gai, K. Zhang, J. Weertman, Scripta Mater. 56 (2007) p. 25.

[33] M. Legros, D.S. Gianola, K.J. Hemker, Acta Mater. 56 (2008) p. 3380.

[34] F. Mompiou, M. Legros, D. Caillard, MRS Symp. Proc. 1086 (2008) p. 1086-U09-04.

[35] S.Brandstetter, K. Zhang, A. Escuadro, J.R. Weertman, H. Van Swygenhoven, Scripta Mater. 58 (2008) p. 61.

[36] A.J. Haslam, D. Moldovan, V. Yamakov, D. Wolf, S.R. Phillpot, H. Gleiter, Acta Mater. 51 (2003) p. 2097.

[37] D. Farkas, A. Frøseth, H. Van Swygenhoven, Scripta Mater. 55 (2006) p. 695.

[38] F. Sansoz, V. Dupont, Appl. Phys. Lett. 89 (2006) p. 111901.

[39] D. Farkas, S. Mohanty, J. Monk, Mater. Sci. Eng. A 493 (2008) 33. 
[40] V. Dupont, F. Sansoz, Acta Mater. 56 (2008) p. 6013.

[41] A.P. Sutton, R.W. Baluffi, Interfaces in Crystalline Materials, Oxford Univ. Press (1995).

[42] Y. Mishin, Int. Workshop on Small Scale Plasticity, Braunwald, Switzerland (2007).

Available at http://smallscalep.web.psi.ch/presentations/Mishin.pdf

[43] S. Van Petegem, F. Dalla Torre, D. Segers, H. Van Swygenhoven, Scripta Mater. 48 (2004) p. 17.

[44] A. Luque, J. Aldazabal, J.M. Martínez-Esnaola, J. Gil Sevillano, Fatigue Fract. Engng. Mater. Struct. 30 (2007) p. 1008.

[45] S. Nosé, J. Chem. Phys. 81 (1984) p. 511.

[46] J. Li, Model. Simul. Mater. Sci. Eng. 11 (2003) p. 173.

[47] W.F. Gale, T.C. Totemeier (eds.), Smithells Metal Reference Book, 8th ed., Elsevier, Amsterdam, 2004, p. 15-5.

[48] R.O.Scattergood, D.J. Bacon, Acta Metall. 30 (1982) p. 1665.

[49] Y.N. Osetski, D.J. Bacon, Mater. Sci. Eng. A 400-401 (2004) p. 374.

[50] A. Luque, J. Aldazabal, J.M. Martínez-Esnaola, J. Gil Sevillano, in preparation.

[51] B. Radiguet, A. Etienne, P. Pareige, X. Sauvage, R. Valiev, J. Mater. Sci. 43 (2008) p. 7338.

[52] L. Zhou, H. Zhang, D.J. Srolowitz, Acta Mater. 53 (2005) 5273.

[53] K. Lücke, H.P. Stüwe, "Recovery and Recrystallization of Metals", L. Himmel (ed.), Interscience, New York, 1963, p.171.

[54] F.J. Humphreys, M. Hatherly, "Recristallization and Related Annealing Phenomena", Pergamon, Oxford, UK, 1996, p. 8.

[55] K.L. Merkle, D. Wolf, Philos. Mag. A 65 (1992) p. 513.

[56] A. Otsuki, Mater. Sci. Forum, 207-209 (1996) p. 413.

[57] M.A. Tschopp, D.L. McDowell, Philos Mag., 87 (2007) p.3147.

[58] M.A. Tschopp, D.L. McDowell, Philos Mag., 87 (2007) p. 3871.

[59] C. Herring, "The Physics of Powder Metallurgy”, W.E. Kingston, ed., McGraw-Hill, New York, 1951, p. 143.

[60] W.K. Burton, N. Cabrera, F.C. Frank, Phil. Trans. A, 243 (1951) p. 299.

[61] G. de Wit, J.S. Koehler, Phys. Rev., 116 (1959) p. 1113.

[61] Y.T. Chou, J. D. Eshelby, J. Mech. Phys. Solids, 10 (1962) p. 27.

[63] L.M. Brown, R.K. Ham, "Strengthening Methods in Crystals", A. Kelly, R.B. Nicholson, eds., Applied Science, London, UK, 1971, p. 9.

[64] A. Moraviec, Scripta Mater., doi:10.1016/j.scriptamat.2009.04.037. 
Table 1. Crystallographic orientation and size of the investigated bicrystals.

\begin{tabular}{|c|c|c|c|c|c|}
\hline $\begin{array}{c}\text { Tilt boundary } \\
\text { designation }\end{array}$ & Tilt axis & $\begin{array}{c}\text { Tilt angle } \boldsymbol{\theta} \\
\mathbf{(}^{\mathbf{o}}\end{array}$ & $\begin{array}{c}\text { Misorientation } \\
\left.\mathbf{(}^{\mathbf{(}}\right)\end{array}$ & $\begin{array}{c}\text { Specimen size, } \\
\boldsymbol{L}_{\mathbf{x}} \times \boldsymbol{L}_{\mathbf{y}} \times \boldsymbol{L}_{\mathbf{z}}\left(\mathbf{n m}^{\mathbf{3}} \mathbf{)}\right.\end{array}$ & $\begin{array}{c}\text { Shear } \\
\text { coupling } \\
\text { factor } \boldsymbol{\beta}\end{array}$ \\
\hline$\Sigma 5(310)$ & {$[001]$} & 36.9 & 36.9 & $13.7 \times 2.9 \times 17.8$ & -1 \\
\hline$\Sigma 17(530)$ & {$[001]$} & 61.9 & 28.1 & $14.8 \times 2.2 \times 21.0$ & -0.5 \\
\hline$\Sigma 41(540)$ & {$[001]$} & 77.3 & 12.7 & $13.9 \times 2.2 \times 18.4$ & -0.222 \\
\hline
\end{tabular}




\section{FIGURE CAPTIONS}

Figure 1. Dislocation glide mechanism assumed for SCM of symmetrical tilt boundaries. Reproduced from ref. [16].

Figure 2. Nominal shear stress, $\tau_{\text {nom }}$, as a function of the nominal shear strain, $\gamma_{\text {nom }}$.

(a) Uncracked or precipitate-free tilt boundaries.

(b) Boundaries with periodic intergranular cracks or precipitates.

Figure 3. SCM of a $\Sigma 17$ symmetrical tilt boundary in copper at room temperature. Two periods of the simulated bicrystal are shown. Colour code: coordination number; atoms with coordination 12 made invisible.

(a) Initial structure.

(b) After macroscopic shear fully resolved by $\operatorname{SCM}\left(\gamma_{\text {nom }}=0.15\right)$.

Figure 4. The $\Sigma 17$ bicrystal with a row of periodic intergranular cracks. Colour code: coordination number; atoms with coordination 12 made invisible.

(a) Initial structure.

(b) After shear deformation $\left(\gamma_{\text {nom }}=0.24\right)$. The macroscopic shear is resolved mainly by SCM, with a small contribution from dislocation glide. Notice that the crack has been healed after the GB depinning from the crack tips.

Figure 5. Shear of a symmetrical $\Sigma 41$ tilt boundary with intergranular cracks. Copper, room temperature. Colour code: coordination number; atoms with coordination 12 made invisible.

(a) Initial structure.

(b) After macroscopic shear $\left(\gamma_{\text {nom }}=0.09\right)$, resolved by SCM plus a contribution from dislocation emission. Some crack healing has occurred.

Figure 6. Shear of a symmetrical $\Sigma 5$ tilt boundary with intergranular cracks. Copper, room temperature. Colour code: coordination number; atoms with coordination 12 made invisible.

(a) Initial structure.

(b) After macroscopic shear $\left(\gamma_{\text {nom }}=0.28\right)$ resolved by grain boundary sliding, GBS. Some crack growth has taken place.

Figure 7. Shear stress-strain behaviour of virtual bicrystals with horizontal boundaries pinned at their free surfaces.

(a) Assuming the GB bulges as a membrane of uniform specific surface energy $E_{S}=1 \mathrm{Jm}^{-2}$ under a pressure $P=\beta\left(\tau-\tau_{c}\right)$.

(b) MD simulation.

Figure 8. Behaviour of bicrystals separated by a tilt grain boundary of any spatial orientation relative to the macroscopic shear plane.

(a) Virtual SCM of a flat tilt boundary inclined with respect to the macroscopic shear system.

(b) Equilibrium shape of a small segment of a curved tilt boundary with an inclination $\alpha$ migrating under a shear stress in excess of the critical stress for 
1

2

3

4

5

6

7

8

9

10

11

12

13

14

15

16

17

18

19

20

21

22

23

24

25

26

27

28

29

30

31

32

33

34

35

36

37

38

39

40

41

42

43

44

45

46

47

48

49

50

51

52

53

54

55

56

57

58

59

60

migration of a flat boundary with the same inclination. The excess stress induces a virtual pressure normal to the boundary surface, eq. (7).

Figure 9. Sketch of the Hall-Petch law breakdown induced by activation of SCM when the yield stress predicted by the Hall-Petch law falls above the critical stress for shearcoupled migration of free flat tilt boundaries. 
Fig. 1

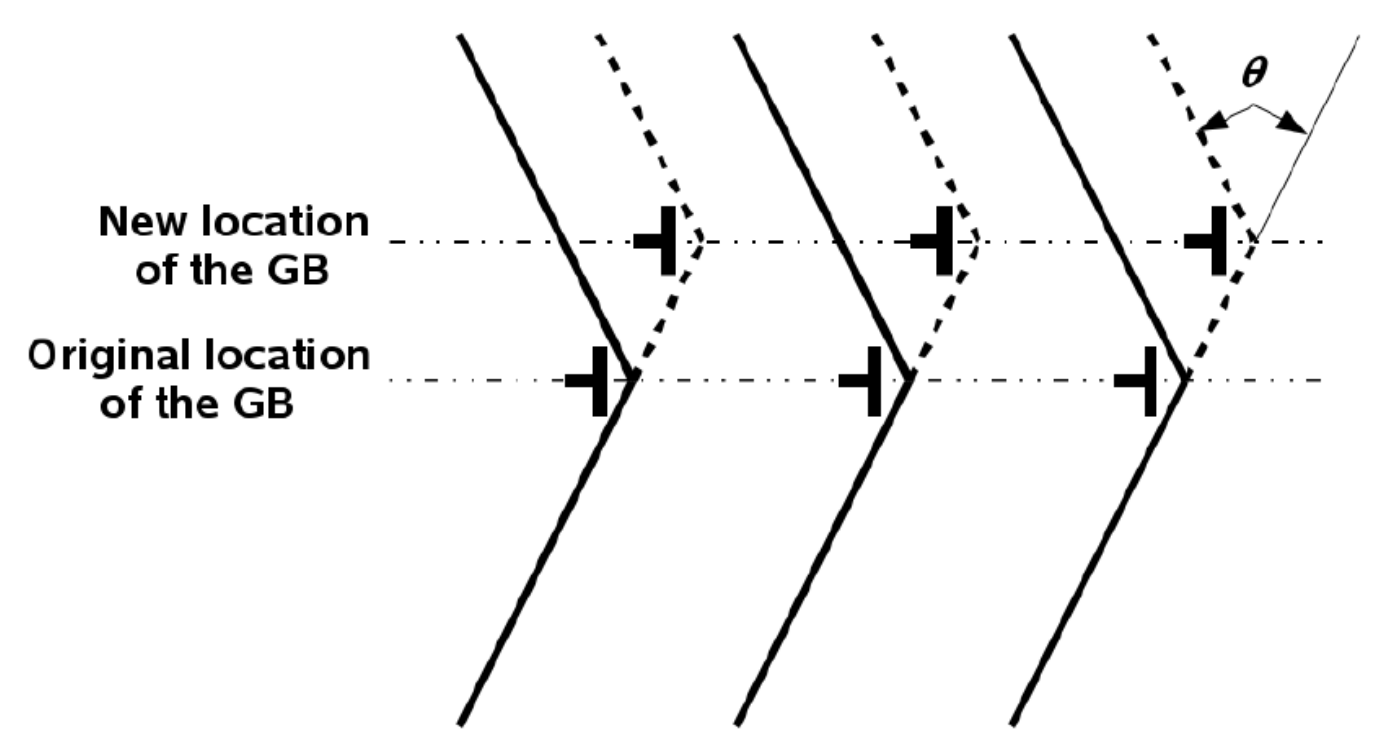




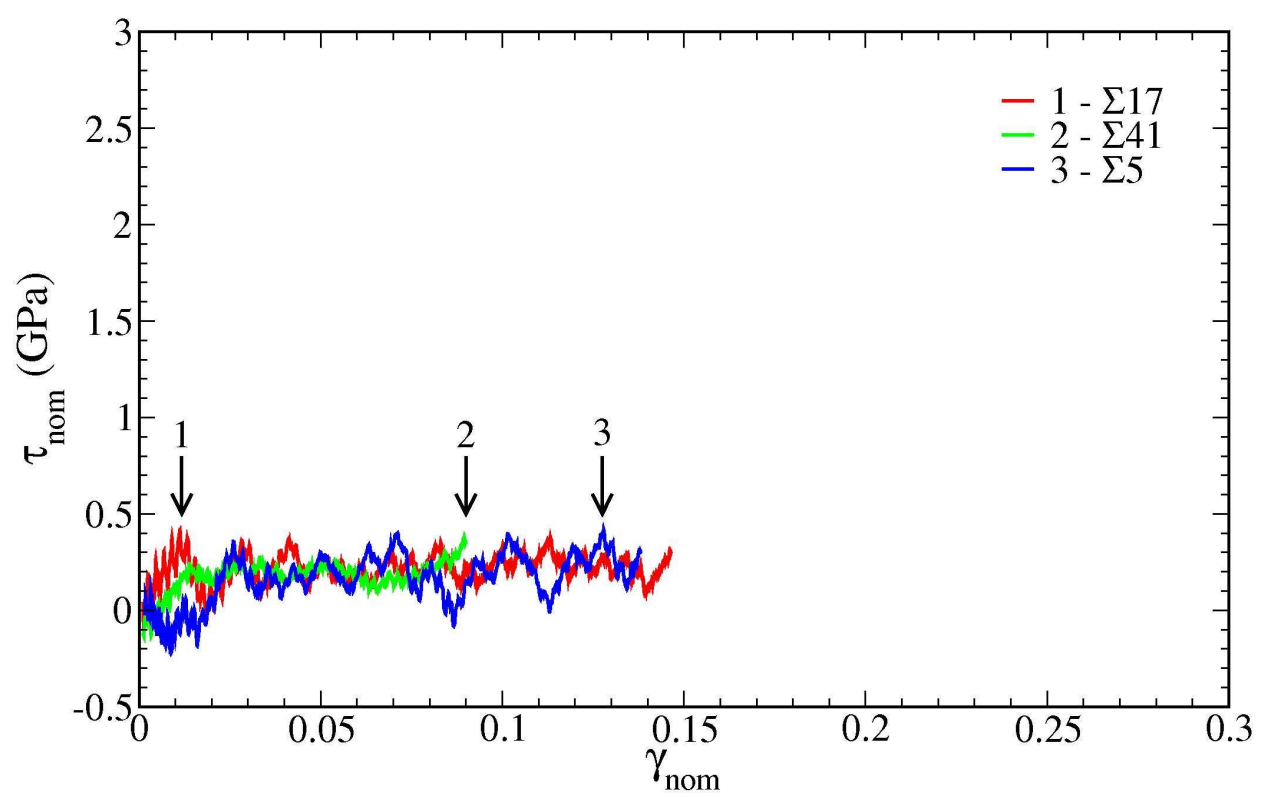

Figure 2. Nominal shear stress, $\tau$ nom, as a function of the nominal shear strain, $\gamma$ nom. (a) Uncracked or precipitate-free tilt boundaries. (b) Boundaries with periodic intergranular cracks or precipitates.

$93 \times 57 \mathrm{~mm}(600 \times 600$ DPI $)$ 


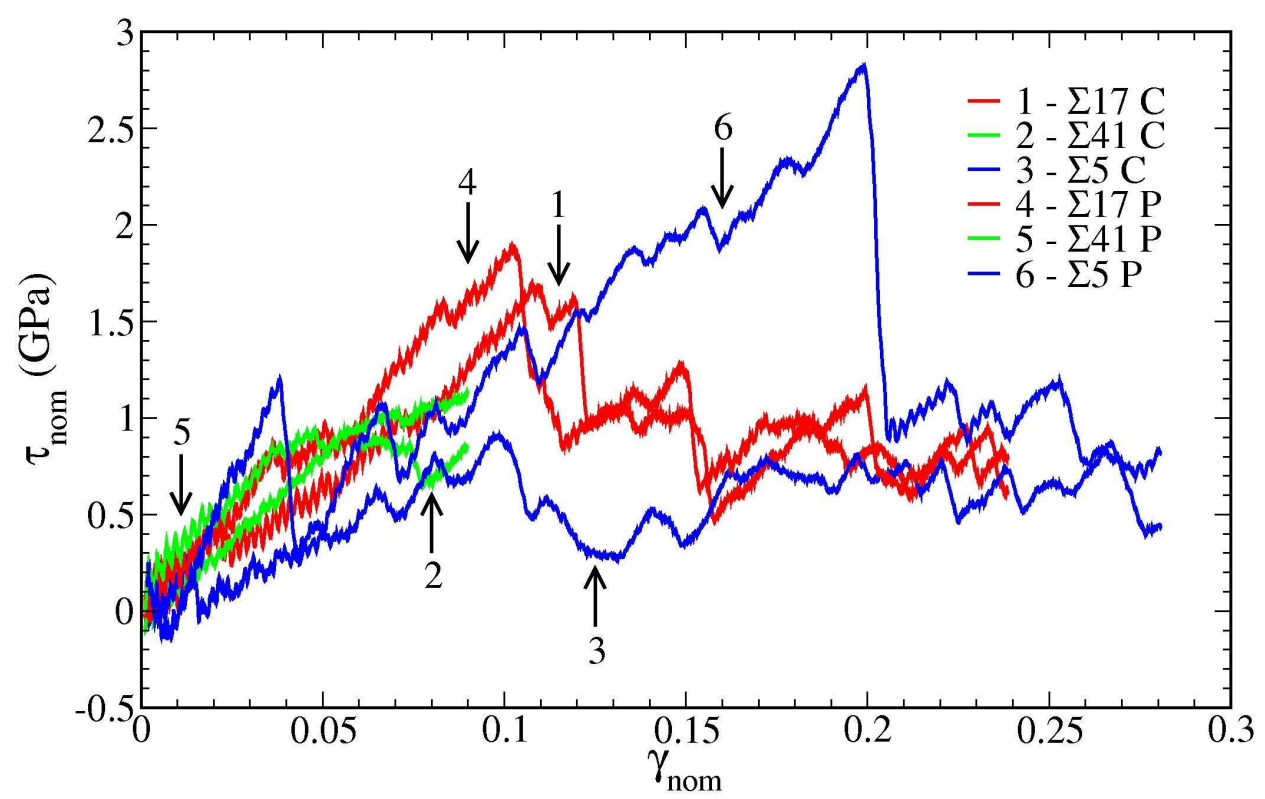

Figure 2. Nominal shear stress, $\tau$ nom, as a function of the nominal shear strain, $\gamma$ nom. (a) Uncracked or precipitate-free tilt boundaries.

(b) Boundaries with periodic intergranular cracks or precipitates.

\section{$93 \times 57 \mathrm{~mm}(600 \times 600$ DPI $)$}


Figure 3. SCM of a $\Sigma 17$ symmetrical tilt boundary in copper at room temperature. Two periods of the simulated bicrystal are shown.

(a) Initial structure.

(b) After macroscopic shear fully resolved by SCM ( ).

$216 \times 163 \mathrm{~mm}(600 \times 600 \mathrm{DPI})$ 
Figure 3. SCM of a $\Sigma 17$ symmetrical tilt boundary in copper at room temperature. Two periods of the simulated bicrystal are shown.

(a) Initial structure.

(b) After macroscopic shear fully resolved by SCM ( ).

$216 \times 163 \mathrm{~mm}(600 \times 600 \mathrm{DPI})$ 
Figure 4. The $\Sigma 17$ bicrystal with a row of periodic intergranular cracks.

(a) Initial structure.

(b) After shear deformation ( ). The macroscopic shear is resolved mainly by SCM, with a small contribution of dislocation glide. Notice that the crack has been healed after the GB depinning from the crack tips.

$216 \times 163 \mathrm{~mm}(600 \times 600 \mathrm{DPI})$ 
Figure 4. The $\Sigma 17$ bicrystal with a row of periodic intergranular cracks.

(a) Initial structure.

(b) After shear deformation ( ). The macroscopic shear is resolved mainly by SCM, with a small contribution of dislocation glide. Notice that the crack has been healed after the GB depinning from the crack tips.

$216 \times 163 \mathrm{~mm}(600 \times 600 \mathrm{DPI})$ 
Figure 5. Shear of a symmetrical tilt boundary with intergranular cracks. Copper, room temperature.

(a) Initial structure.

(b) After macroscopic shear resolved by SCM ( ) and a contribution from dislocation emission. Some crack healing has occurred.

$216 \times 163 \mathrm{~mm}(600 \times 600 \mathrm{DPI})$ 


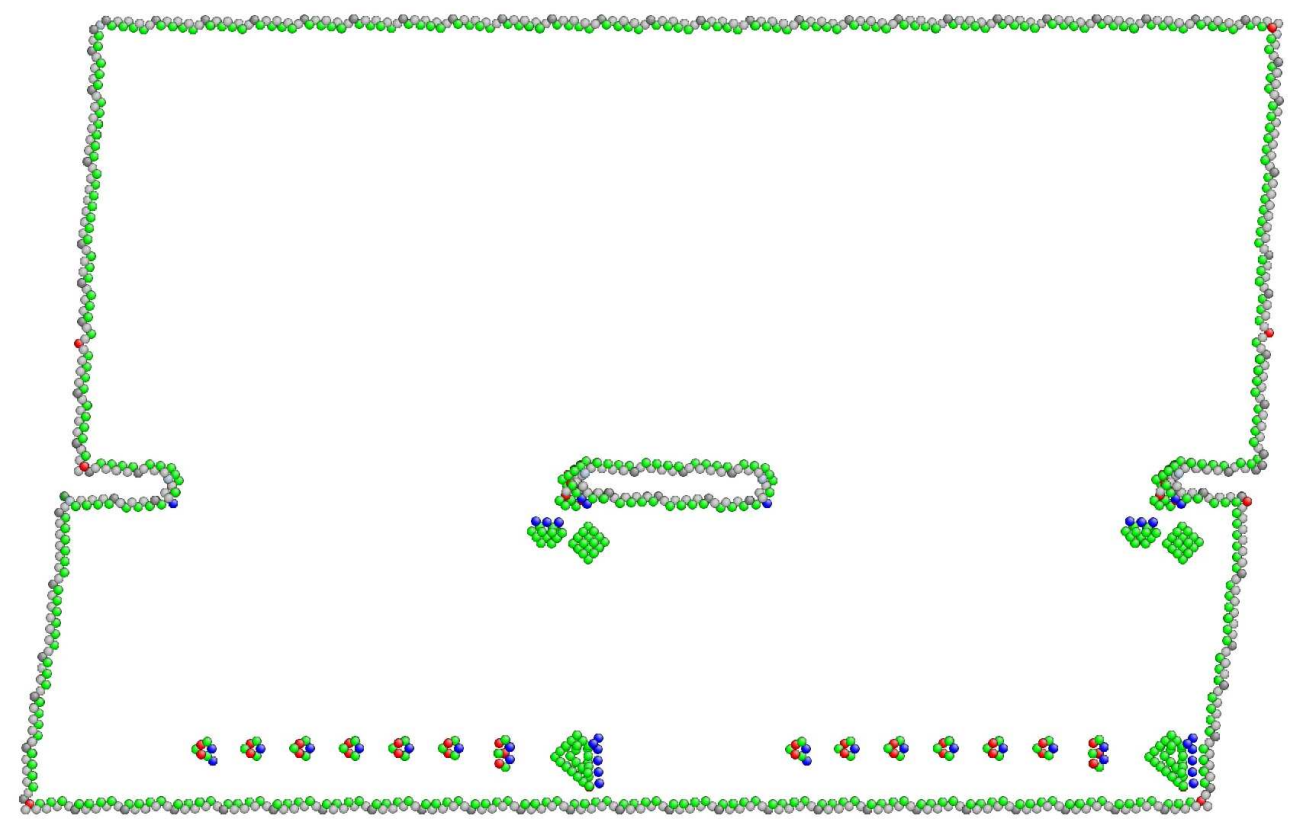

Figure 5. Shear of a symmetrical tilt boundary with intergranular cracks. Copper, room temperature.

(a) Initial structure.

(b) After macroscopic shear resolved by SCM ( ) and a contribution from dislocation emission. Some crack healing has occurred.

$216 \times 163 \mathrm{~mm}(600 \times 600 \mathrm{DPI})$ 
Figure 6. Shear of a symmetrical tilt boundary with intergranular cracks. Copper, room temperature.

(a) Initial structure.

(b) After macroscopic shear ( ) resolved by grain boundary sliding, GBS. Some crack growth has taken place.

$216 \times 163 \mathrm{~mm}(600 \times 600 \mathrm{DPI})$ 


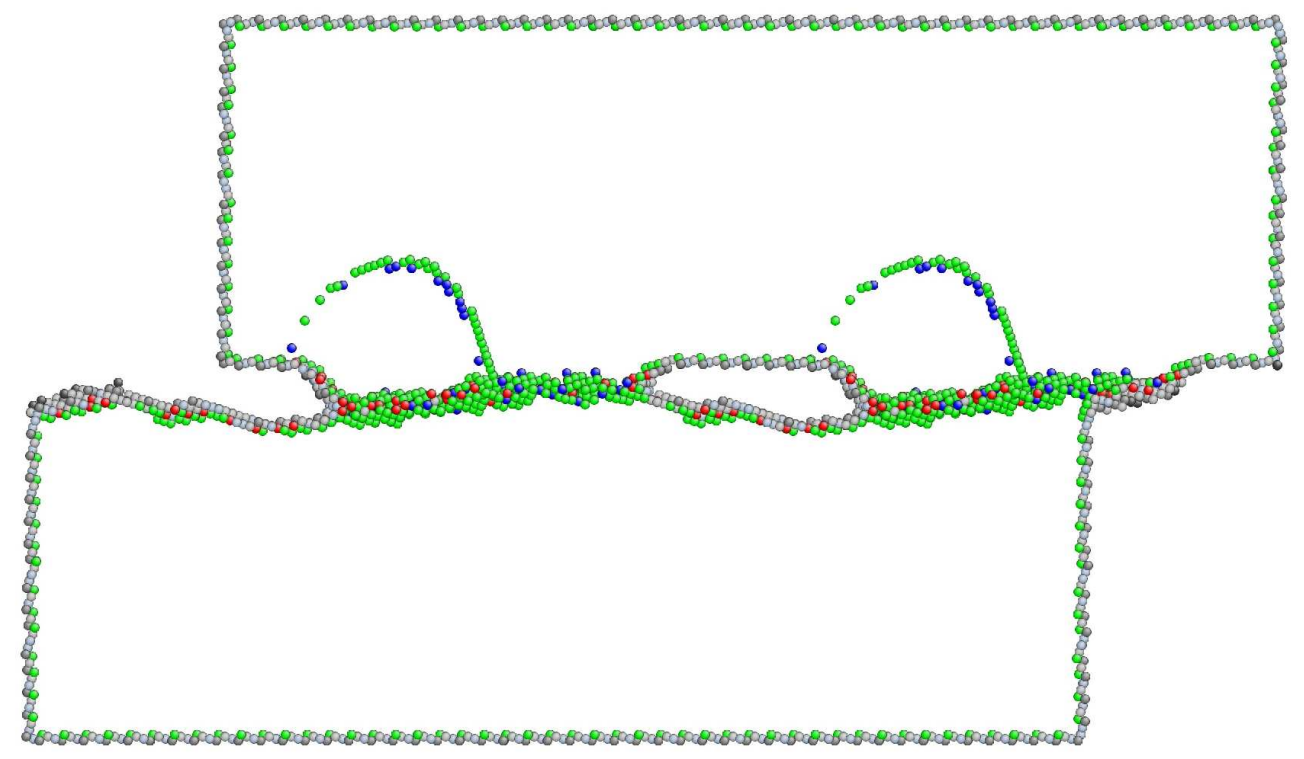

Figure 6. Shear of a symmetrical tilt boundary with intergranular cracks. Copper, room temperature.

(a) Initial structure.

(b) After macroscopic shear ( ) resolved by grain boundary sliding, GBS. Some crack growth has taken place.

$216 \times 163 \mathrm{~mm}(600 \times 600 \mathrm{DPI})$ 
Figure 7. Shear stress-strain behaviour of virtual bicrystals with horizontal boundaries pinned at their free surfaces.

(a) Assuming the GB bulges as a membrane of uniform specific surface energy Jm-2 under a pressure .

(b) MD simulation.

$93 \times 57 \mathrm{~mm}(600 \times 600 \mathrm{DPI})$ 


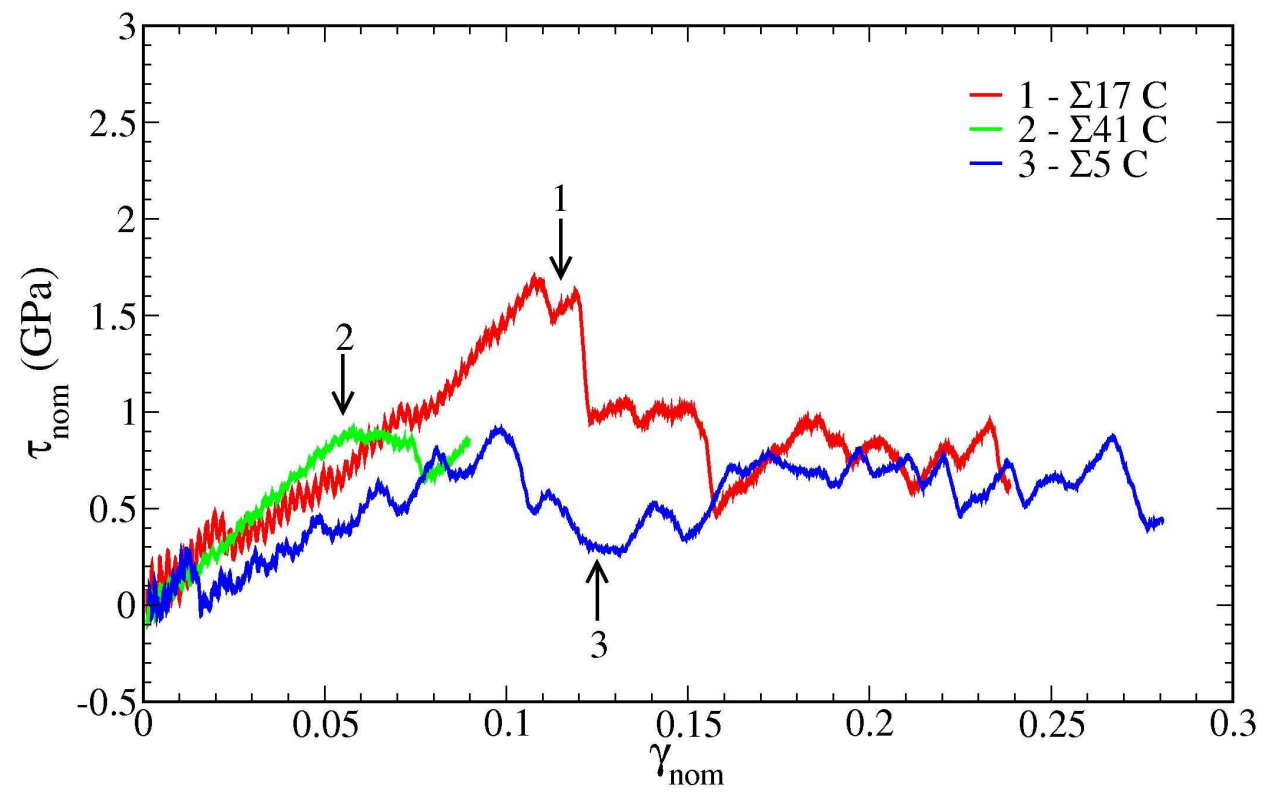

Figure 7. Shear stress-strain behaviour of virtual bicrystals with horizontal boundaries pinned at their free surfaces.

(a) Assuming the GB bulges as a membrane of uniform specific surface energy Jm-2 under a pressure.

(b) MD simulation.

$93 \times 57 \mathrm{~mm}(600 \times 600 \mathrm{DPI})$ 
Figure 8a

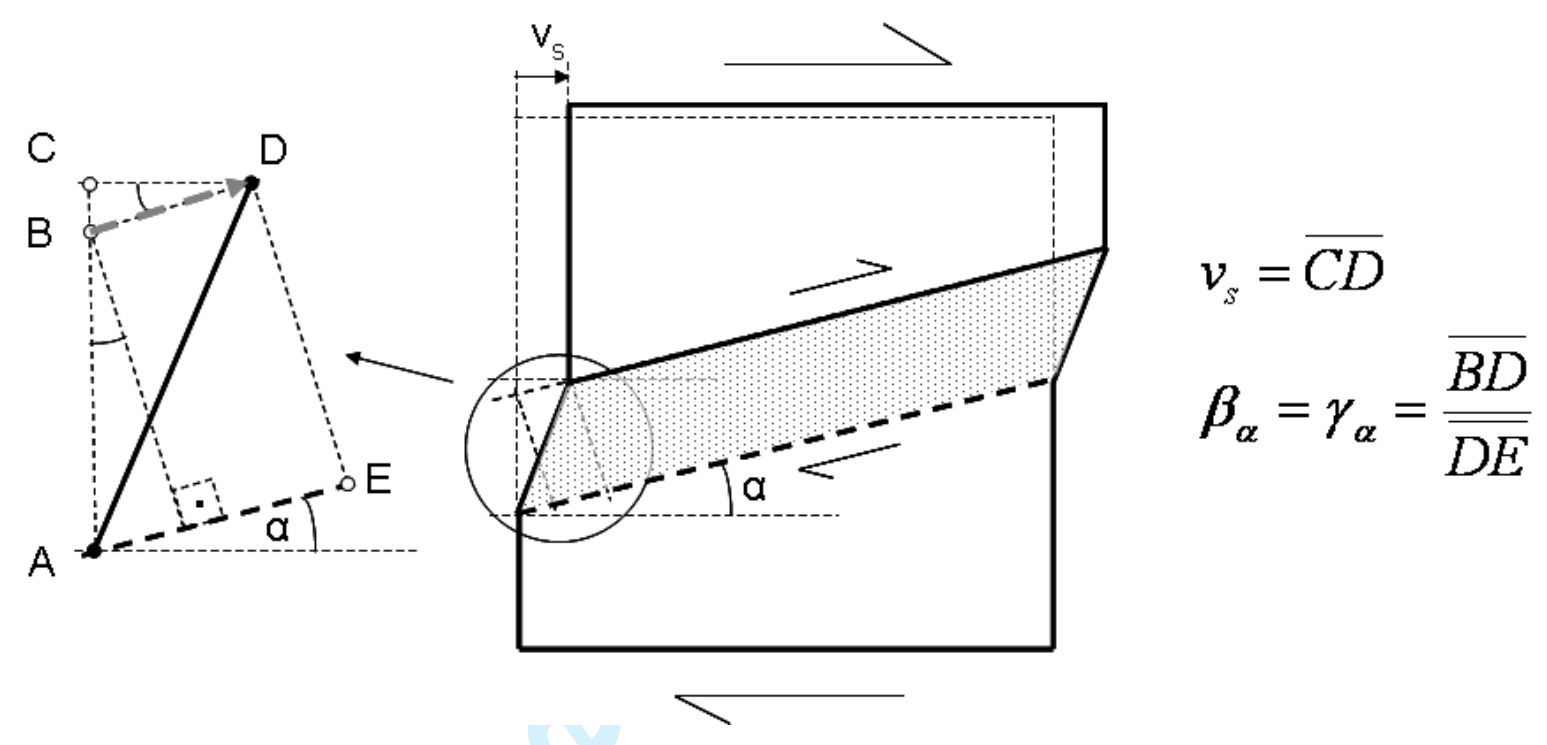

Figure $8 b$

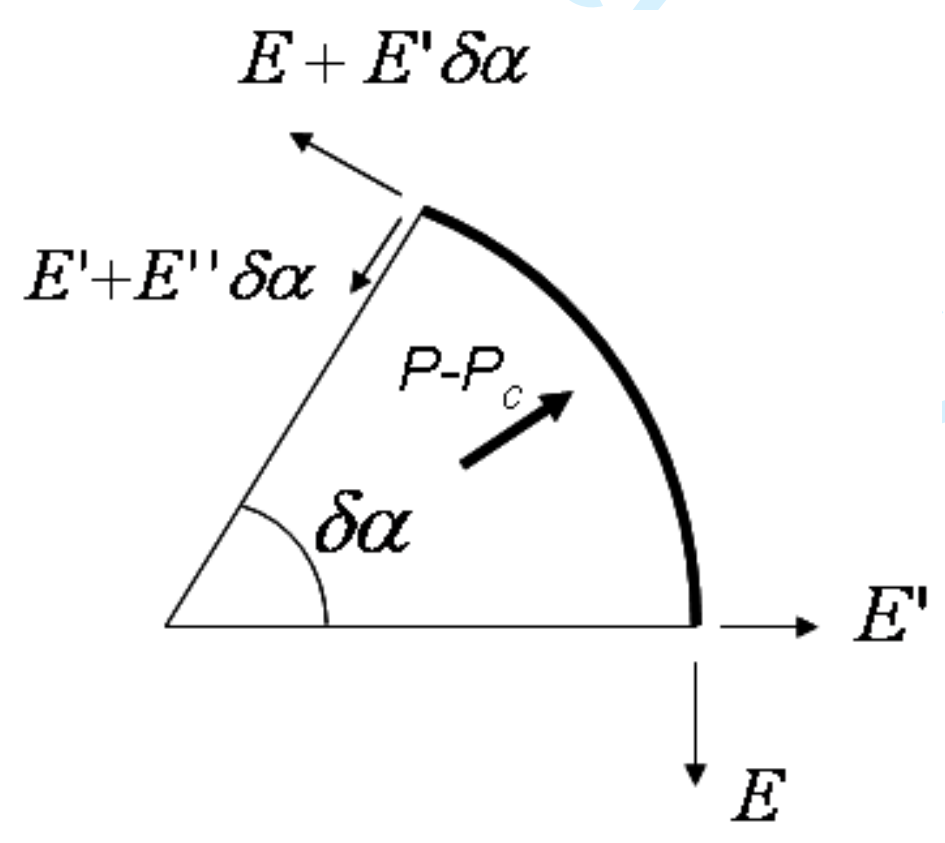




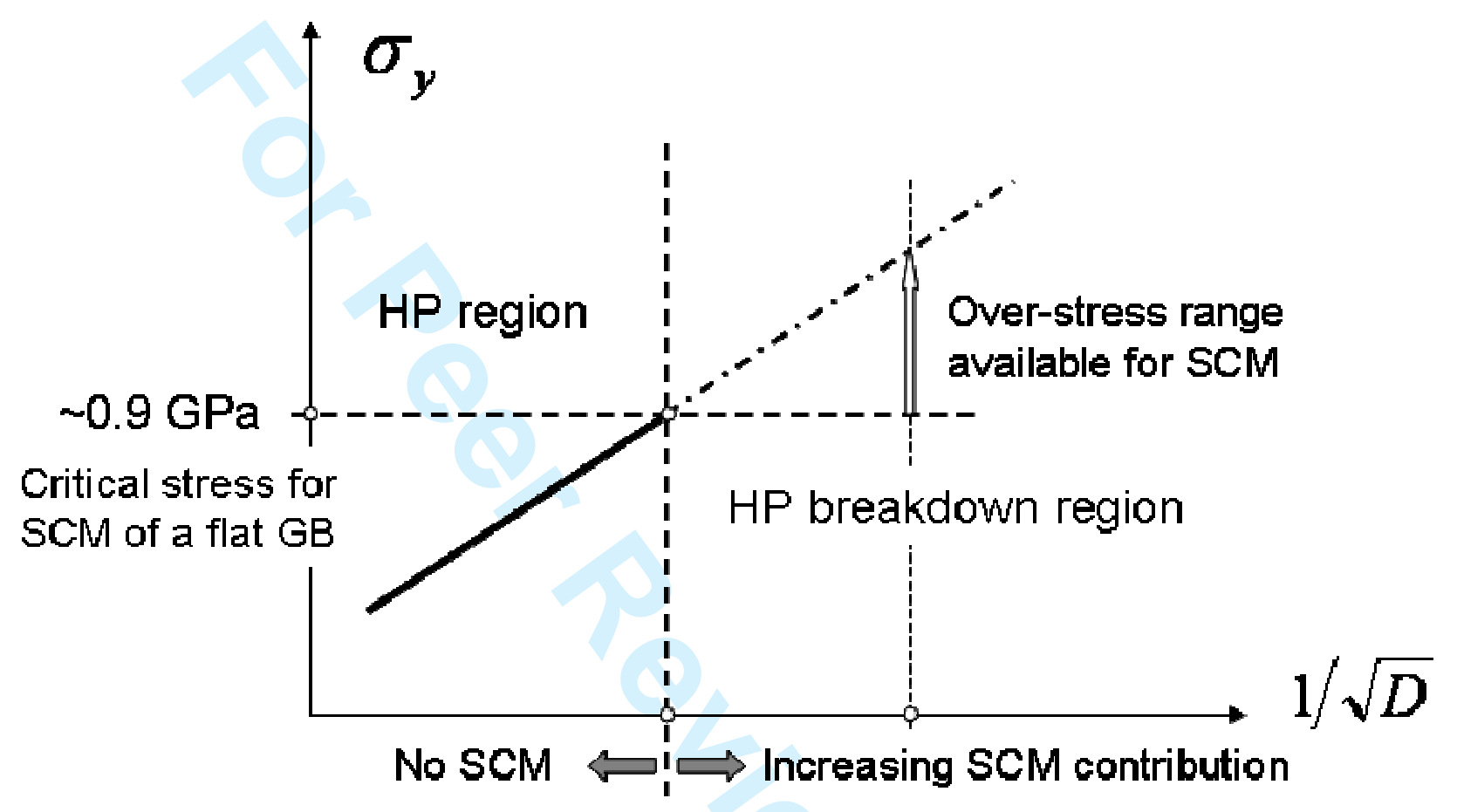

Figure 9 